\title{
Rigidity in Condensed Matter and Its Origin in Configurational Constraint
}

\author{
Shibu Saw and Peter Harrowell \\ School of Chemistry, University of Sydney, Sydney NSW 2006 Australia
}

(Dated: October 14, 2018)

\begin{abstract}
Motivated by the formal argument that a non-zero shear modulus is the result of averaging over a constrained configurations space, we demonstrate that the shear modulus calculated over a range of temperatures and averaging times can be expressed (relative to its infinite frequency value) as a single function of the mean squared displacement. This result is shown to hold for both a glass-liquid and a crystal-liquid system.
\end{abstract}


Glasses are rigid and liquids are not. The difficulty with any distinction of two phases based solely on rigidity is that the property is not an equilibrium one. Over 45 years ago, Lebowitz[1] and Ruelle[2] pointed out that, in the thermodynamic limit, the free energy of a phase cannot depend on the shape of the sample and so the equilibrium value of the shear modulus must vanish for all phases - crystals as well as glasses - in the limit of large N. Rationalising the obvious point that rigid materials do in fact exist, a number or researchers [3] have concluded that a non-zero shear modulus is a property of a metastable state and hence rigidity is observable only for observation times shorter than the lifetime of that state. Since the observation of a non-zero shear modulus depends crucially on this lifetime, it would seem that any theoretical treatment of the mechanical properties of a material will depend on solving the onerous problem of slow relaxation in a condensed phase. Williams and Evans[4], acknowledging this difficulty, suggested that the shear modulus be formally calculated as an equilibrium average over a constrained space of configurations. This perspective suggests the attractive possibility that the magnitude of the shear modulus might be expressed as an explicit function of the magnitude of the configurational constraint applied, a relation that includes a threshold degree of constraint, below which rigidity vanishes. In this paper we establish just such a relationship between the shear modulus and the configurational constraint, measured here by the mean squared displacement, for both a glass-liquid and crystal-liquid system.

The Squire-Holt-Hoover expression [5] for the (constrained) equilibrium shear modulus $G_{e q}$ of a solid is

$$
G_{e q}=G_{\infty}-\beta V\left[<\sigma^{2}>-<\sigma>^{2}\right]
$$

where $\sigma$ is the shear stress, $\beta=1 / k_{B} T, V$ is volume and $G_{\infty}$ is the infinite frequency (or Born) shear modulus given by[6]

$$
G_{\infty}=\frac{N}{V} k_{B} T-\frac{1}{2 V} \sum_{i} \sum_{j \neq i}<\left(y_{i j}^{2} F_{i j}\left[1-\frac{x_{i j}^{2}}{r_{i j}^{2}}\right]-\frac{d^{2} \phi}{d r_{i j}^{2}} \frac{x_{i j}^{2} y_{i j}^{2}}{r_{i j}^{2}}\right)>
$$

where $F_{i j}=-\frac{1}{r_{i j}} \frac{d \phi}{d r_{i j}}$ and $\phi(r)$ is a spherically symmetric inter-particle potential. Note that the shear modulus $G_{e q}$ is reduced, relative to the high frequency value, by an amount associated with variance of the shear stress fluctuations. In the context of elastic theory, these fluctuations correspond to non-affine contributions to the modulus[7]. What is measured in 
a typical experiment is the stress relaxation function $G(t)=\sigma(t) / \gamma$, where $\gamma$ is a applied strain and $\sigma(t)$ is the resulting time dependent shear stress. The relation between $G(t)$ and $G_{e q}$ is given by the following expression[8],

$$
G(t)=G_{e q}+\beta V\left[<\sigma(0) \sigma(t)>-<\sigma>^{2}\right]
$$

where the shear stress autocorrelation function $<\sigma(0) \sigma(t)>$ equals $<\sigma^{2}>$ when $\mathrm{t}=0$ and $\langle\sigma\rangle^{2}$ in the limit $t \rightarrow \infty$. It follows from Eq. 3 that $G_{e q}$ represents a lower bound to the observed modulus $G(t)$ with $\lim _{t \rightarrow \infty} G(t)=G_{e q}$. This long time limit refers only to the explicit time dependence arising from the shear stress autocorrelation function. It does not include any implicit time dependence associated with the observation time used to construct the averages in $G_{e q}$ (see Eq. 1). So, the averages $<\ldots>$ in Eq. 1-3 are understood to be taken over some constrained configuration space. In the absence of a constraint, $\langle\sigma\rangle=0$ and $G_{\infty}=\beta V<\sigma^{2}>[9]$ so that $G_{e q}=0$.

The model liquid used in this study is a 2D system of soft disks with a pair interaction potential, $\phi_{i j}(r)=\epsilon\left(\frac{a_{i j}}{r}\right)^{12}$, between species $i$ and $j$. In the case of the binary equimolar mixture we use $a_{11}=1.0, a_{22}=1.4$ and $a_{12}=1.2$ and all particle with unit mass, a model that has been extensively studied [10] in the context of the glass transition. The temperature is reported in units of $\epsilon / k_{B}$ and time in units of $\tau=\sqrt{m a_{11}^{2} / \epsilon}$. Simulations were carried out under constant NVT conditions using LAMMPS[11] with a Nose-Hoover thermostats at reduced densities 0.7468 (binary mixture) and 1.398 (single component) with a potential cut-off distance of $6.3 a_{11}$. The system consisted of a total of $\mathrm{N}=1024$ particles in the case of the binary mixture and $N=1400$ for the single component system. Previously[12], we established that these values of $\mathrm{N}$ were sufficient for accurate calculation of the stress fluctuations. At low temperatures, the trajectories are non-ergodic for all accessible values of the averaging time $t$. In order to fairly sample the configuration space at these low temperatures we have averaged trajectories over statistically distinct initial configurations. For the binary mixtures, 51 uncorrelated configurations were generated by cooling a liquid, equilibrated at $T=0.60$, to $T=0.30$ at a cooling rate of $5 \times 10^{-5}$ and then minimizing the potential energy of the resulting $T=0.30$ liquid by conjugate gradient minimization. The minima, referred to as inherent structures (IS), were statistically independent as established by the average shear stress (at $T=0$ ) of the inherent structures equalling zero. To calculate $G_{e q}$ and $G_{\infty}$ at a given temperature $T$ we randomly assigned momenta from the Boltzmann 

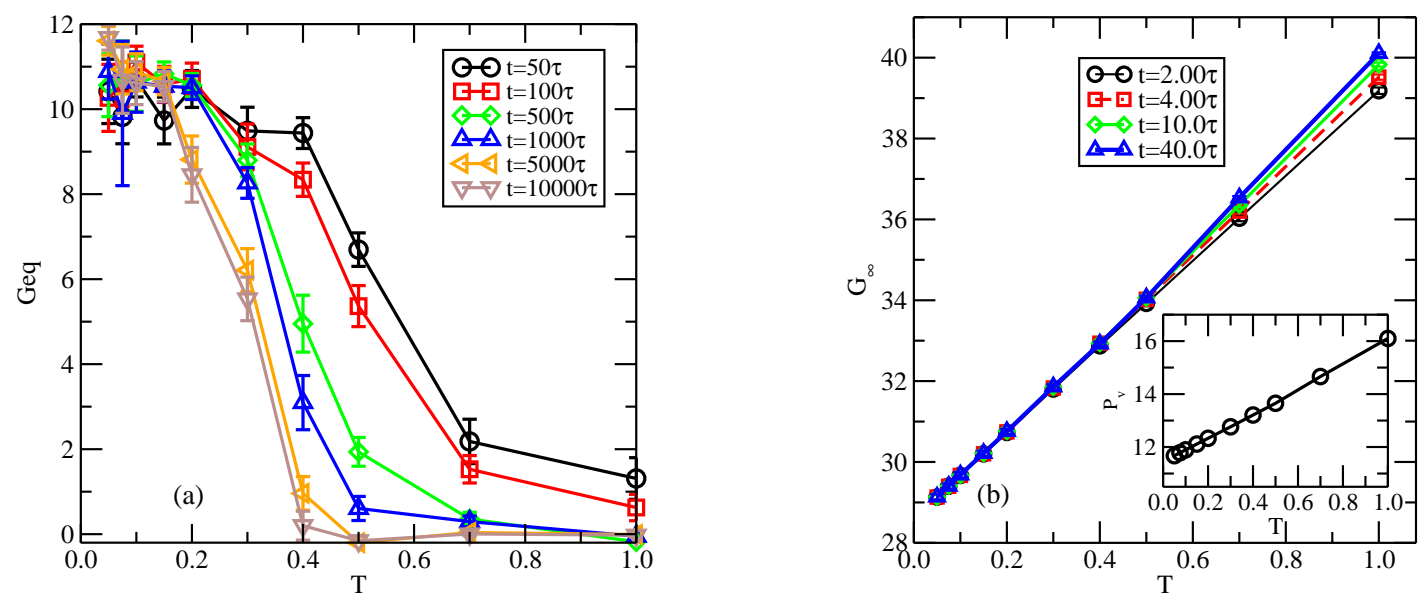

FIG. 1: (Color online) Plot of (a) $G_{e q}$ and (b) $G_{\infty}$ vs $T$ for the binary mixture over different averaging times. Note the significant effect of the averaging time in the case of $G_{e q}$ in contrast to $G_{\infty}$, were the influence of averaging time has saturated within a short time $\sim 40 \tau$. Insert in Fig. 1b: The virial pressure $P_{\mathrm{v}}=-\frac{1}{2 V}<\sum_{i} \sum_{j \neq i} r_{i j} \frac{d \phi}{d r_{i j}}>$ as a function of $T$.

distribution consistent with a temperature $T$ to the particles in each of the $\mathrm{n}$ IS configurations and then determined the moduli for each individual IS using Eqs. 1and 2, respectively, by averaging over a trajectory run for a time interval t. To obtain our final values of $G_{e q}$ and $G_{\infty}$, we averaged the moduli for the individual IS configurations over all 51 IS configurations. In the case of the single component system, this protocol was modified as follows. For the crystal phase we only used a single inherent structure, that of the perfect crystal. For the liquid phase data we simply carried out averages over MD trajectories of the equilibrated liquid.

In Fig. 1 we plot the values of $G_{e q}$ and $G_{\infty}$ as a function of $T$ for a binary mixture of soft disks in $2 D$ at a fixed density. We find that $G_{\infty}$ increases linearly with $T$ and shows no significant variation with the averaging time t. (Note that the infinite frequency modulus referred to experimentally is not $G_{\infty}$ but the value of $G(t)$ in the plateau region.) The equilibrium modulus $G_{e q}$, in contrast, exhibits a strongly nonlinear decrease with increasing temperature, to finally vanish at a sufficiently high temperature. The family of curves presented in Fig. 1 is evidence of the significance of the time $t$ used to calculate the statistics of the stress fluctuations. 
A number of papers [13 15] have discussed the loss of rigidity of a glass as characterised by the disappearance of $G_{e q}$ on heating in the context of a possible thermodynamic instability, analogous to the softening in a superheated crystal[13, 14], or as an un-jamming transition associated with the thermal expansion of the amorphous solid[15]. (We remind the reader that our calculations have been carried out at fixed density so that this latter proposal is not directly relevant here.) While the role of the observation time is discussed[14], it is the temperature that is treated as the essential control parameter for the transition. Following on from our opening discussion, we shall explore the idea that this decrease of the equilibrium shear modulus, either through increasing $\mathrm{T}$ or the observation time, is most fundamentally expressed as a result of the changing degree of configurational constraint associated with the averaging.

To begin we note that temperature dependence of $G_{\infty}$ is not associated with configurational constraint since, by construction, the infinite frequency modulus depends only on the sampling of the local curvature of the potential energy surface. The increase in $G_{\infty}$ with temperature at constant volume demonstrated in Fig. 1b is directly associated with the increase of the virial pressure with $\mathrm{T}$ under the constant volume constraint (see insert Fig. 1b). To eliminate this additional temperature dependence we shall therefore consider the reduced modulus $G_{e q} / G_{\infty}$. Next, we need a measure of the configurational constraint. The simplest such measure is the particle mean squared displacement,

$$
<\Delta r^{2}(t)>=\frac{1}{N} \sum_{i}<\left|\vec{r}_{i}(0)-\vec{r}_{i}(t)\right|^{2}>
$$

where the time $t$ here is the same as the observation time used to calculate the stress averages and, in the case of a binary mixture, the average is over both species.

In Fig. 2 we plot $G_{e q} / G_{\infty}$ vs $\left\langle\Delta r^{2}>\right.$ where we have used the data from Fig. 1 for a range of temperatures and observation times. We find that all of the data from Fig. 1 collapses onto a single curve. This result provides strong support the twin propositions of this paper, i.e. that the (reduced) shear modulus is simply a consequence of configurational constraint and that the mean squared displacement provides a useful measure of this constraint.

A glass forming liquid is convenient for our purposes because it can access the entire range of $G_{e q}$ without encountering a thermodynamic singularity. Our argument relating shear mod- 


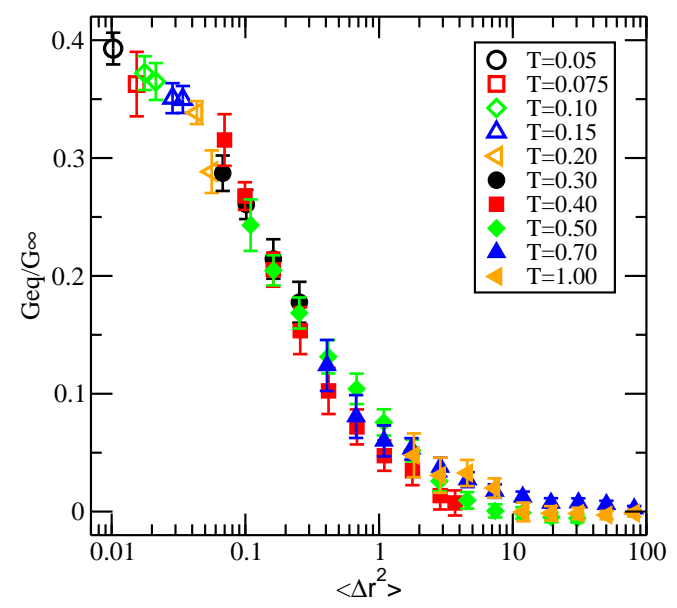

FIG. 2: (Color online) Plot of $G_{e q} / G_{\infty}$ vs $<\Delta r^{2}>$ for the binary mixture. In each case, the mean squared displacement is calculated over the same time interval as that use to evaluate $G_{e q}$.

ulus and configurational constraint, however, should apply equally to crystallizing liquids. To demonstrate this point, we consider a single component soft disk liquid in $2 \mathrm{D}$ which crystallizes readily into a triangular lattice. In Fig. 3 we plot the values of $G_{e q}$ and $<\Delta r^{2}>$ for the system as a function of $T$, using an observation time $t=199 \tau$. The presence of

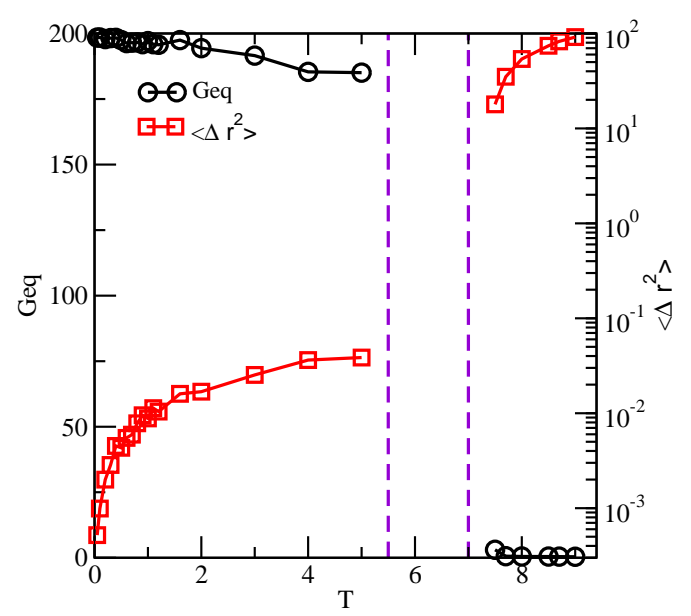

FIG. 3: (Color online) The temperature dependence of $G_{e q}$ and $<\Delta r^{2}>$ (both averaged over a time $199 \tau$ ) for the single component 2D soft disk system. Due to the constant density constraint, there is a range of temperatures corresponding to a two phase coexistence, indicated by the two vertical dashed lines. The freezing transition is marked by step-like change in the modulus and the mean squared displacement. 
the $1^{\text {st }}$ order freezing at $T=5.0$ is clearly evident in both quantities. In Fig. 4 we plot $G_{e q} / G_{\infty}$ vs $<\Delta r^{2}>$ for a range of temperatures (using crystal and liquid configurations for $T$ below and above $T_{m}$, respectively) and a range of observation times. Again, we find the data collapsed onto a common curve, this in spite of the discontinuity of the modulus and $\left\langle\Delta r^{2}\right\rangle$ with respect to temperature. It is worth noting the striking difference in the low $T$ limit of $G_{e q} / G_{\infty}$ for the crystal (Fig. 4) and the glass (Fig. 2). The reason for the considerable softening of the glass relative to $G_{\infty}$ even at $T=0$ is due to (i) the higher density of crystal and (ii) the large non-affine motions[7] in the glass relative to those in the crystal. The presence of non-affine motions in the amorphous phase and their effective absence in the crystal is due to the absence of inversion symmetry in the local structure of the amorphous phase and its presence in the crystal[16].

The dependence of the reduced shear modulus $G_{e q} / G_{\infty}$ on $<\Delta r^{2}>$ is found, empirically, to be well described by the following relation,

$$
\frac{G_{e q}}{G_{\infty}}=\left.\frac{G_{e q}}{G_{\infty}}\right|_{T=0.05} \exp \left(-q \ln ^{\alpha}\left[\frac{<\Delta r^{2}>}{<\Delta r^{2}>\left.\right|_{T=0.05}}\right]\right)
$$

The success of this function is shown in Fig. 5 for the glass forming mixture with the fitted values $\alpha=2$ and $q=0.08$. Eq. 5 also provides an excellent fit to $G_{e q} / G_{\infty}$ vs $<\Delta r^{2}>$ for both the crystal and liquid phases of the single component system (see Fig. 4), but with different parameters, $\alpha=2.9$ and $q=0.0061$. Viewed as an emergent property of restricted particle fluctuations, the derivation of the dependence of $G_{e q} / G_{\infty}$ on the degree of configurational constraint must represent a problem of fundamental importance.

We have argued here that the value of $G_{e q}$ (relative to $G_{\infty}$ ) is a consequence of constraint. This is the opposite to the account provided within harmonic models of solids in which the elastic constants (or the bond force constants) are prescribed in the model and the mean squared displacement are determined as a consequence. This latter treatment, however, is only possible because of the implicit configurational constraints (i.e. assumed elasticity, unbreakable harmonic bonds, etc.) on which such models rely. For the harmonic solid, $G_{e q}<\Delta r^{2}>/ T=$ constant (at fixed density). As shown in Fig. 5 (insert), this relation holds only for $<\Delta r^{2}><0.1$, a result that underscores the inclusion of anharmonic effects in the empirical relations demonstrated in Fig. 4 and 5. Yoshino and Zamponi[17] have 


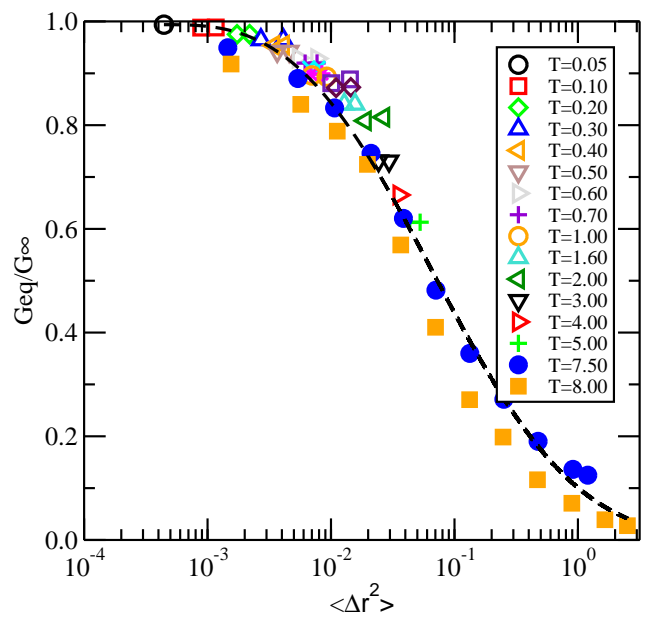

FIG. 4: (Color online) The dependence of $G_{e q} / G_{\infty}$ on $\left\langle\Delta r^{2}\right\rangle$ for the single component crystal and liquid. As for Fig. 2, each point corresponds to a choice of $T$ and the averaging time. Liquid state data i.e. $T>5.0$ are presented by filled symbols and crystal data by open symbols. The curve corresponds of a fit of Eq. 5 to the single component data with $\alpha=2.9$ and $q=0.0061$.

recently derived a power law relationship between the shear modulus and the mean squared displacement in a granular model that applies within a metabasin, a restricted range of configuration space corresponding, roughly, to $0.01 \leq<\Delta r^{2}>\geq 0.2$.

In conclusion, we have verified that our two propositions: (1) the degree of configurational constraint determines the magnitude of the shear modulus (relative to the low temperature limit), and (2) the mean squared displacement provides a useful measure of this constraint, do indeed represent a consistent physical picture for both a glass forming liquid and one that undergoes freezing. This result represents a fundamental unification of the physical basis of rigidity. The presence of a non-zero shear modulus is not, we argue, the consequence of a low temperature, a high frequency measurement or even the presence of long range order. Rather, each of these factors is important only in as far as they contribute to an implicit constraint on the volume of configuration space that can be explored by stress fluctuations. It is this constraint, however it is achieved, that determines the value of the equilibrium shear modulus. This is a powerful result with a number of interesting consequences. First, accounts of the temperature dependence of the shear modulus of metallic glasses [18] have relied on the language of anharmonic effects borrowed from crystal physics. In the picture 
we present here, the decrease in the glass modulus on heating is associated the increase in $<\Delta r^{2}>$ by harmonic or anharmonic motions (along with any decrease in $G_{\infty}$ associated with thermal expansion when a constant pressure is employed as in ref. [14] ). Second, we have argued that the shear modulus should be regarded as a mechanical manifestation of restricted motion. Couple this idea with a description of the role that elastic behaviour plays in determining the rate of particle motion (e.g. the shoving model of Dyre[19]) and there is possibility of a self consistent theory in which the modulus is, itself, a consequence of the very particle mobilities that it acts to constrain. Thirdly, these results suggest a reassessment of the empirical Lindemann criterion[20], i.e. the observation that crystal order is lost once the mean squared displacement exceeds some threshold value. Our results here suggest that it is rigidity, not structure per se, that vanishes as the mean square displacement increases. Finally, since our account of rigidity places no special condition on how the configuration space is accessed it is possible that non-thermal contributions to particle mobility such as the non-affine motion due to applied strain should result in an analogous reduction in $G_{e q} / G_{\infty}[21]$. Each of these lines of inquiry is currently under investigation.

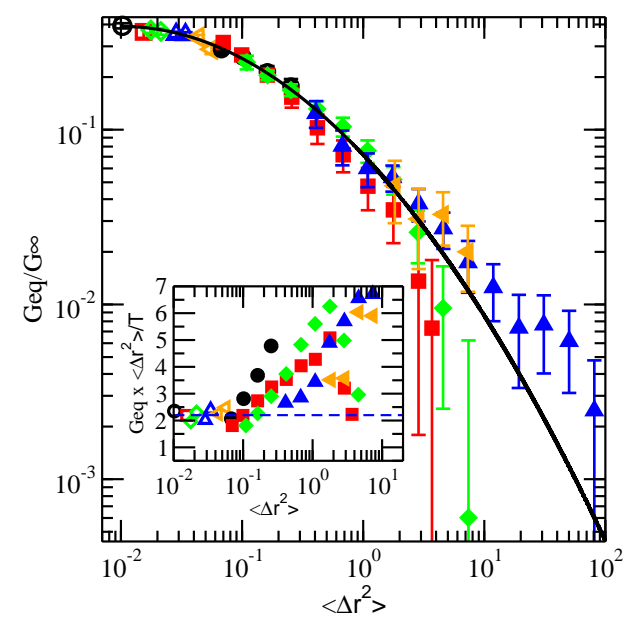

FIG. 5: (Color online) A log-log plot of of $G_{e q} / G_{\infty}$ vs $<\Delta r^{2}>$ for the binary mixture. (The correspondence between symbols and temperatures are the same as in Fig. 2). The expression in Eq. 5 (solid curve) provides a good description over the entire range of $\left\langle\Delta r^{2}>\right.$ with $\alpha=2.0$ and $q=0.08$. Insert: The quantity $G_{e q}<\Delta r^{2}>/ T$ vs $<\Delta r^{2}>$. The harmonic approximation, indicated by a constant value (dashed line), breaks down for $<\Delta r^{2}>\geq 0.1$. 
In this paper we have established that the collection of factors - time, temperature and order - associated with the observation of rigidity in a dense phase can be replaced by a single tangible length that characterizes the degree of configurational constraint. While we have established that $\left\langle\Delta r^{2}>\right.$ provides a workable measure of this constraint length, further work is required to establish whether there is a better measure of this constraint and whether we can derive from first principles the mathematical relationship between this measure and the shear modulus.

\section{ACKNOWLEDGEMENTS}

We acknowledge support from the Australian Research Council.

\section{REFERENCES}

[1] J. Lebowitz, Ann. Rev. Phys. Chem. 19, 389-418 (1968).

[2] D. Ruelle, Statistical Mechanics. Rigorous Results (World Scientific, London 1999).

[3] J. Frenkel, Kinetic Theory of Liquids (Oxford University Press, London, 1946); F. Sausset, G. Biroli and J. Kurchan, J. Stat. Phys. 140, 718-727 (2010).

[4] S. R. Williams and D. J. Evans, J. Chem. Phys. 131, 024115(1-9) (2009); ibid, J. Chem. Phys. 132, 184105(1-14) (2010); S. R. Williams, J. Chem. Phys. 135, 131102(1-4) (2011).

[5] D. R. Squire, A. C. Holt and W. G. Hoover, Physica 42, 388-397 (1969).

[6] I. Fuereder and P. Ilg, J. Chem. Phys. 142, 144505 (2015).

[7] A. Lemaitre and C. Maloney, J. Stat. Phys. 123, 415- 453 (2006).

[8] H. Yoshino, J. Chem. Phys. 136, 214108(1-36) (2012).

[9] R. Zwanzig and R. D. Mountain, J. Chem. Phys. 43, 4464-4471 (1965).

[10] D. N. Perera, and P. Harrowell, Phys. Rev. E 59, 5721-5743 (1999).

[11] S. Plimpton, J. Comp. Phys. 117, 1-19 (1995); http://lammps.sandia.gov.

[12] S. Abraham and P. Harrowell, J. Chem. Phys. 137, 014506(1-8) (2012).

[13] J. -L. Barrat, J.-N. Roux, J.-P. Hansen and M. L. Klein, Europhys. Lett. 7, 707-712(1988). 
[14] J. P. Wittmer, H. Xu, P. Polinska, F. Weysser and J. Baschnagel, J. Chem. Phys. 138, 12A533(1-18) (2013); J. P. Wittmer, H. Xu, O. Benzerara and J. Baschnagel, Mol. Phys. 113, 2881-2893 (2015).

[15] A. Zaccone and E. M. Terentjev, Phys. Rev. Lett. 110, 178002(1-5) (2013).

[16] A. Zaccone, J. R. Blundell and E. M. Terentjev, Phys. Rev. B 84, 174119 (2011).

[17] H. Yoshino and F. Zamponi, Phys. Rev. E 90, 022302(1-14) (2014).

[18] Y. P. Mitrofanov, V. A. Khonik, A. V. Granato, D. M. Joncich and S. V. Khonic, J. App. Phys. 109, 073518(1-4) (2011).

[19] J. C. Dyre, Rev. Mod. Phys. 78, 953- 972 (2006).

[20] F. A. Lindemann, Phys. Z. 11, 609 (1910).

[21] S. Saw and P. Harrowell, cond-mat arXiv:1602.02855. 